\title{
Preparation and Characterization of Pure Oligoethylene Glycols I.
}

\author{
Shinichi Kinugasa, Hisao Hayashi,* and Shigeru Hattori \\ National Chemical Laboratory for Industry, \\ Higashi, Tsukuba 305, Japan \\ * Department of Materials Chemistry, Ryukoku University, \\ Seta, Otsu 520-21, Japan \\ (Received May 16, 1990)
}

\begin{abstract}
The solution properties of a pure oligoethylene glycol, i.e., octadecaethylene glycol (OEG18), in acetonitrile have been studied by light scattering and small-angle X-ray scattering (SAXS) measurements. No strong downturn at small angles have been observed in the Zimm plot of light scattering data, indicating that OEG18 molecules disperse molecularly in solution. The weight-average molecular weight $M_{w}(=833)$ obtained by light scattering measurements is in good agreement with the expected molecular weight $M(=810.9)$ of OEG18. The radius of gyration $\left\langle S^{2}\right\rangle^{1 / 2}(=9.6 \pm 0.4 \AA)$ has been determined by SAXS, yielding the characteristic ratio $C_{n}=4.8 \pm 0.4$. KEY WORDS Poly(ethylene oxide)/Oligoethylene Glycol / Light Scattering

/ Small-Angle X-Ray Scattering / Radius of Gyration / Characteristic Ratio /
\end{abstract}

Poly(ethylene oxide) (PEO) is a simple polymer with unusual properties. ${ }^{1}$ One of the characteristic features of PEO is that the polymer tends to aggregate in various solvents. Elias and Lys ${ }^{2}$ pointed out that PEO aggregates in some solvents and light scattering data show a strong downturn at small angles in Zimm plots for PEO in methanol, ${ }^{3}$ dimethylformamide, ${ }^{4}$ and water, ${ }^{5}$ indicating the presence of some aggregates in these good solvents.

To discuss unperturbed chain dimensions of polymers, the characteristic ratio $C_{n}=$ $\left\langle R^{2}\right\rangle_{0} / n \bar{l}^{2}$ is often used, where $\left\langle R^{2}\right\rangle_{0}$ is the unperturbed mean-square end-to-end distance, $n$ the number of skeletal bonds, and $\bar{l}^{2}$ the average of the square of their length. ${ }^{6}$ The value of $C_{n}$ can be estimated from the unperturbed mean-square radius of gyration $\left\langle S^{2}\right\rangle_{0}$ (= $\left\langle R^{2}\right\rangle_{0} / 6$ for large $n$ ) obtained by a scattering method such as light scattering, small-angle X-ray scattering (SAXS), and small-angle neutron scattering (SANS). However, few reliable scattering experiments have been carried out for characterizing conformational properties of PEO molecule in solutions. Consequently, $C_{n}$ for PEO has been discussed on the basis of the intrinsic viscosity $[\eta]$ data accumulated for various solvents. ${ }^{7}$

In our previous study, we described a method for preparing a pure oligoethylene glycol, i.e., octadecaethylene glycol (OEG18). ${ }^{8}$ It is of interest to determine characteristic parameters such as the molecular weight $M$ of OEG 18 by light scattering, because a compound of definite molecular weight can be used as a standard sample for calibrating light scattering instruments. ${ }^{9}$ Additionally, a series of pure PEO samples such as OEG18 can also be used as ideal molecular weight standards; for example, in gel permeation chromatography (GPC), calibration curves between molecular weights of PEO and elution volumes will be established more precisely by using these pure samples than by commercial standard PEOs. In the present study, we measured light scattering and SAXS from OEG18 in acetonitrile. Particularly, 
SAXS gives scattered intensities for large values of scattering vector $q$ and therefore, is a useful technique for determining the radius of gyration $\left\langle S^{2}\right\rangle^{1 / 2}$ of oligomers such as OEG18.

\section{EXPERIMENTAL}

\section{Materials}

Pure OEG18 was prepared according to the procedures of Booth et al. ${ }^{10}$ using the reaction between the $\alpha, \omega$-ditosylate and the monosodium salt of hexaethylene glycol (Aldrich Chemical Co.). Separation of the OEG18 from the reaction mixture of oligomeric glycols $(x=6,12,18$, etc.) was carried out by applying the preparative scale GPC. The IR, ${ }^{1} \mathrm{H}$ and ${ }^{13} \mathrm{C}$ NMR spectra obtained for the OEG18 were found to be consistent with those expected for the structure of OEG18. The experimental details were described in our previous paper. ${ }^{8}$ In Figure 1, an analytical GPC chromatogram for the pure OEG18 sample is illustrated together with the one for a commercial PEG standard sample.

Spectral grade acetonitrile (DOJINDO Lab. Co., Japan) was distilled and used as a solvent for light scattering measurements. For SAXS measurements, the solvent was used without purification. Spectral grade benzene (DOJINDO Lab. Co.) was distilled and used for calibrating the light scattering apparatus. Solutions of OEG18 in acetonitrile were prepared gravimetrically and heated at $50^{\circ} \mathrm{C}$ in sealed tubes for 8 hours to remove traces of aggregates or crystallites.

\section{Light Scattering}

Light scattering measurements were carried out with a commercial photometer DLS-700 (Otsuka Electronics Col., Ltd., Japan). A vertically polarized light of $\lambda_{0}=633 \mathrm{~nm}$ wavelength from a $\mathrm{He}-\mathrm{Ne}$ laser was used as an incident beam. Intensities of scattered light were measured at scattering angles from $\theta=30^{\circ}$ to $130^{\circ}$. A cylindrical cell with $21 \mathrm{~mm}$ outer diameter was placed in a thermostated

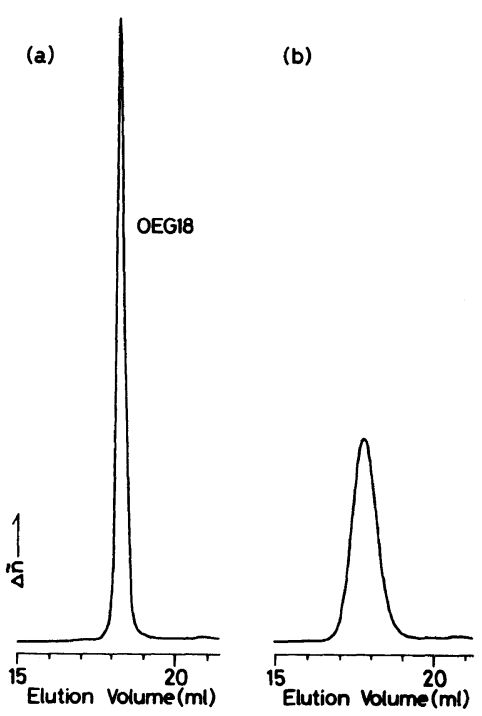

Figure 1. Analytical GPC chromatograms for (a) pure OEG18, and (b) a commercial PEO standard sample (nominal $M=960, M_{w} / M_{n}=1.07$ ). Same conditions for both chromatograms: columns, TSKgel-G3000 $\mathrm{H}_{\mathrm{xL}} \times 2$; flow rate, $1 \mathrm{ml} \mathrm{min}^{-1}$; eluent, THF; injection of samples, $0.18 \mathrm{mg}$.

$\left(25.0 \pm 0.1^{\circ} \mathrm{C}\right)$ bath filled with di- $n$-butyl phtalate. The sample solutions and solvents were optically purified by filtration through a PTFE membrane filter with a nominal pore size of $0.2 \mu \mathrm{m}$ (Toyo Roshi Co., Japan). The photometer was calibrated with benzene and NBS-705 polystyrene (nominal $M_{w}=1.793 \times$ $10^{5}$ ) as the standard. Scattering intensities from benzene were constant within $1 \%$ from $30^{\circ}$ to $130^{\circ}$ after scattering volume correction. Measurements on NBS-705 polystyrene in benzene gave reasonable values of the weight-average molecular weight $M_{w}$, the second virial coefficient $A_{2}$, and the $z$-average radius of gyration $\left\langle S^{2}\right\rangle^{1 / 2}$. To calculate absolute Rayleigh ratios for the sample solutions, we took the Rayleigh ratio of pure benzene as $R_{\mathrm{B}}=$ $11.84 \times 10^{-6} \mathrm{~cm}^{-1} \cdot{ }^{11}$

\section{Refractive Index Increment}

Specific refractive index increment $\partial \tilde{n} / \partial c$ of OEG18 in acetonitrile was measured at $\lambda_{0}=633 \mathrm{~nm}$ using a Brice type differential 
refractometer KMX-16 (Chromatix Inc.). The measured value was $\partial \tilde{n} / \partial c=0.130 \mathrm{~cm}^{3} \mathrm{~g}^{-1}$ at $25^{\circ} \mathrm{C}$. For the refractive index $\tilde{n}_{\mathrm{s}}$ of acetonitrile at $\lambda_{0}=633 \mathrm{~nm}$, we used $\tilde{n}_{\mathrm{s}}=1.3403$ calculated from the values at $\lambda_{0}=436$ and $546 \mathrm{~nm}$ by Couchy dispersion formula. ${ }^{12}$

\section{Small-Angle X-Ray Scattering}

SAXS measurements were performed at $25^{\circ} \mathrm{C}$ with a point-focusing SAXS camera at the High Intensity X-ray Laboratory of Kyoto University. ${ }^{13}$ The X-ray generator equipped to RU-1000 (Rigaku Co., Japan) was operated at $2.4 \mathrm{~kW}$ for the $\mathrm{Cu}$ target $\left(\lambda_{0}=1.54 \AA\right)$. Sample to detector distance was $1627 \mathrm{~mm}$ and the detector was a two dimensional position sensitive proportional counter with an active area of $128 \times 128 \mathrm{~mm}^{2}$ with resolution of $1.0 \times 0.5 \mathrm{~mm}^{2}$. Two dimensional data of scattered intensities were corrected for detector sensitivity, background scattering due to the solvent and the sample cell, and transmission. To obtain the scattered intensity as a function of the scattering angle, corrected scattering data were circularly averaged, because scattering patterns are isotropic azimutally. The details of the SAXS instrument were described by Hayashi et al. ${ }^{13}$

\section{RESULTS AND DISCUSSION}

We used acetonitrile as a solvent for PEO in the scattering experiments, because a molecular weight of a PEO sample determined by light scattering in acetonitrile has been found to agree with GPC values. ${ }^{5}$ Typical examples of angular dependence of scattering intensity are presented for three solutions, including those at the lowest and highest concentrations, in Figure 2, where the inverse of the excess Rayleigh ratio $R(q)$ is plotted against the square of the scattering vector $q$ (= $\left.\left(4 \pi \tilde{n}_{\mathrm{s}} / \lambda_{0}\right) \sin (\theta / 2)\right)$ instead of $\sin ^{2} \theta / 2$. As Figure 2 shows, the data points become more scattered with decreasing concentration; even at the highest concentration, its scattered intensities

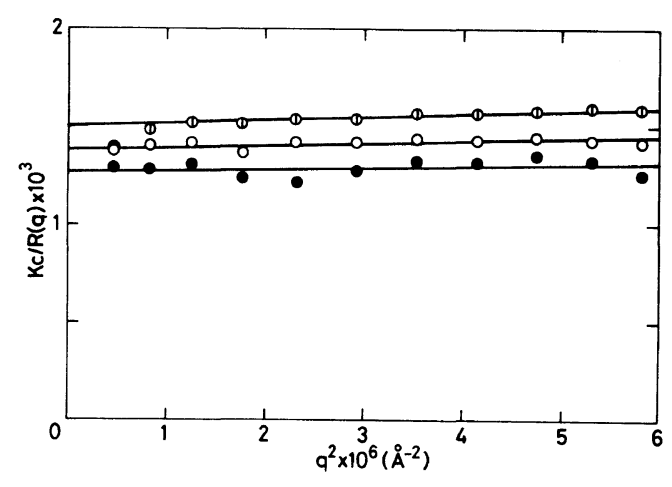

Figure 2. Examples of the plot of reciprocal Rayleigh ratio $K c / R(q)$ against the square of the scattering vector $q$ for OEG18 in acetonitrile at $25^{\circ} \mathrm{C}$. The sample concentrations $c$ are (O) 0.01303 , (O) 0.03698 , and (D) $0.06050 \mathrm{~g} \mathrm{ml}^{-1}$, respectively.

are only about two times larger than those of the solvent.

Recent light scattering experiments show that a strong downturn is observed in the Zimm plots at small angles for PEO in various solvents such as water $^{5}$ and methanol. ${ }^{3}$ Contrary to these experimental findings, our data points in Figure 2 show no strong downturn at small angles. This behavior of $K c / R(q)$ indicates the OEG18 molecules are molecularly dispersed in the solutions. However, a careful inspection of the plots indicates slight slopes of the straight lines for each concentration. Furthermore, a slight downturn is observed in data for the highest concentration. We are not certain whether this slope is caused by the presence of large particles such as aggregates or crystallites in the solution.

In Figure 3, examples of angular dependence of the excess scattered intensity $\Delta I(q)$ obtained for SAXS are given by the plot of $(c / \Delta I(q))^{1 / 2}$ against $q^{2}$ for OEG18 in acetonitrile at $25^{\circ} \mathrm{C}$. From the slopes of the straight lines in the Guinier region $\left(q<1 /\left\langle S^{2}\right\rangle^{1 / 2}\right)$, apparent values of $\left\langle S^{2}\right\rangle_{z, \text { ap }}^{1 / 2}$ were evaluated at each concentration. The concentration dependence of $\left\langle S^{2}\right\rangle_{z, \text { ap }}^{1 / 2}$ thus obtained is shown in Figure 4 . As generally observed for polymer solutions, $\langle S\rangle_{z \text {,ap }}^{1 / 2}$ decreases with increasing concentration $c$. The 


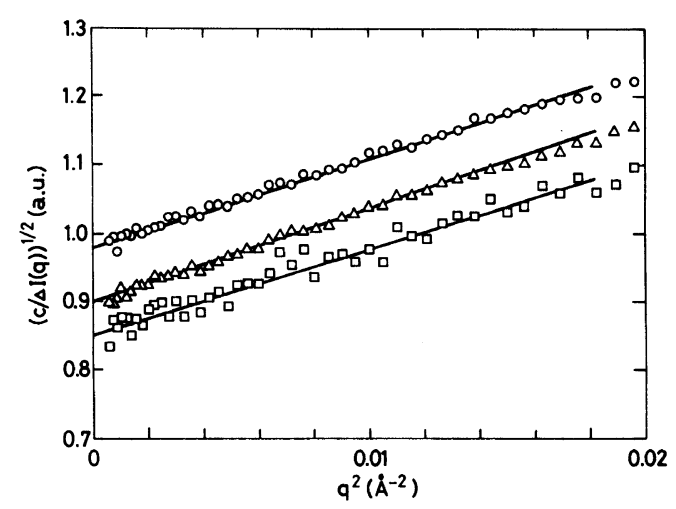

Figure 3. Examples of reciprocal square root of the excess scattered intensity $\Delta I(q)$ as a function of $q^{2}$ for OEG18 in acetonitrile at $25^{\circ} \mathrm{C}$. The sample concentrations $c$ are $(\square) 0.02313,(\triangle) 0.08941$, and $(\bigcirc) 0.1330 \mathrm{~g} \mathrm{ml}^{-1}$, respectively.

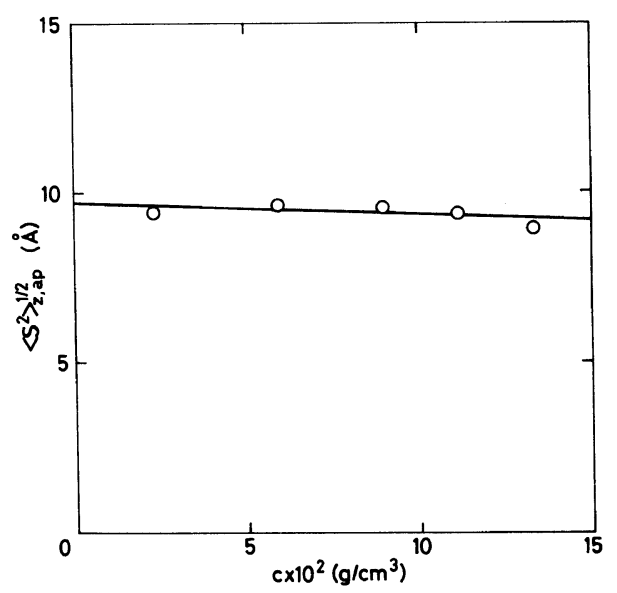

Figure 4. Concentration dependence of apparent radius of gyration $\left\langle S^{2}\right\rangle_{z, \text { ap }}^{1 / 2}$ for OEG18 in acetonitrile at $25^{\circ} \mathrm{C}$. The straight line was obtained by the least-squares method and its intercept at $c=0$ gives the values of $\left\langle S^{2}\right\rangle^{1 / 2}=$ $9.7 \AA$.

data, though somewhat scattered, are fitted by a straight line and extrapolated to zero concentration $(c \rightarrow 0)$ to evaluate $\left\langle S^{2}\right\rangle^{1 / 2}$. The value of $\left\langle S^{2}\right\rangle^{1 / 2}$ thus obtained is $9.7 \pm$ $0.4 \AA$.

As will be described later in the present paper, the second virial coefficient $A_{2}$ of OEG18 in acetonitrile has a positive value, indicating the polymer chain is perturbed by the excluded-volume effect. Nevertheless, we can regard the observed $\left\langle S^{2}\right\rangle^{1 / 2}$ as the unperturbed radius of gyration $\left\langle S^{2}\right\rangle_{0}^{1 / 2}$, because the molecular weight of OEG18 is so small that the excluded-volume effect should vanish. According to the idea of the critical onset of the excluded-volume effect, ${ }^{14,15}$ it is expected that there exists a critical molecular weight $M_{\mathrm{e}}$ for each polymer below which the effect practically vanishes even in good solvents. By using the molecular parameters of the helical wormlike (HW) chain model, ${ }^{16}$ we estimated $M_{\mathrm{e}}$ for PEO; $M_{\mathrm{e}} \simeq 600$ from Yamakawa et al.'s results $^{15}$ and $M_{\mathrm{e}} \simeq 6000$ from Norisuye and Fujita's analysis, ${ }^{14}$ respectively. Taking into account the ambiguity of $M_{\mathrm{e}}$, the molecular weight of OEG18 satisfies $M \leqq M_{\mathrm{e}}$ and, therefore, the oligomer is practically under the unperturbed or the $\Theta$-condition.

According to Konishi et al.'s procedure, ${ }^{17}$ appropriate correction for the finite cross section of the polymer chain must be taken into account to estimate the radius of gyration for the chain contour from the data of $\left\langle S^{2}\right\rangle^{1 / 2}$ determined by SAXS measurements. On the basis of their analysis, we estimated the radius of gyration $S_{\mathrm{c}}$ for the cross section using the HW cylinder model with diameter $d$, where $d=2\left(\bar{v} M_{\mathrm{L}} / \pi N_{\mathrm{A}}\right)^{1 / 2}$ with $\bar{v}=0.851 \mathrm{~cm}^{3} \mathrm{~g}^{-118}$ the partial specific volume of $\mathrm{PEO}$ in acetonitrile and $M_{\mathrm{L}}=8.8 \AA^{-1}$ its shift factor. ${ }^{16}$ The estimated value of $d$ is $3.98 \AA$, yielding $S_{\mathrm{c}}=1.41 \AA$. Using this value of $S_{\mathrm{c}}$, we obtained the corrected radius of gyration $\left\langle S^{2}\right\rangle^{1 / 2}=9.6 \AA$ for the chain contour, which is slightly smaller $(0.1 \AA)$ than that determined by the SAXS experiment.

The characteristic ratio $C_{n}$ for OEG 18 was calculated from the radius of gyration $\left\langle S^{2}\right\rangle^{1 / 2}$ obtained above. We obtained $C_{n}=4.8 \pm 0.4$ by substituting the values $\left\langle S^{2}\right\rangle^{1 / 2}=9.6 \AA, n=54$ and $l^{2}=2.14 \AA^{2}$ into the following equation

$$
C_{n}=6\left\langle S^{2}\right\rangle_{0} / n T^{2}
$$

where $\bar{l}^{2}=\left(l_{\mathrm{C}-\mathrm{C}}^{2}+2 l_{\mathrm{C}-\mathrm{o}}^{2}\right) / 3$ with $l_{\mathrm{C}-\mathrm{C}}$ the length of the $\mathrm{C}-\mathrm{C}$ skeletal bond and $l_{\mathrm{C}-\mathrm{O}}$ that 


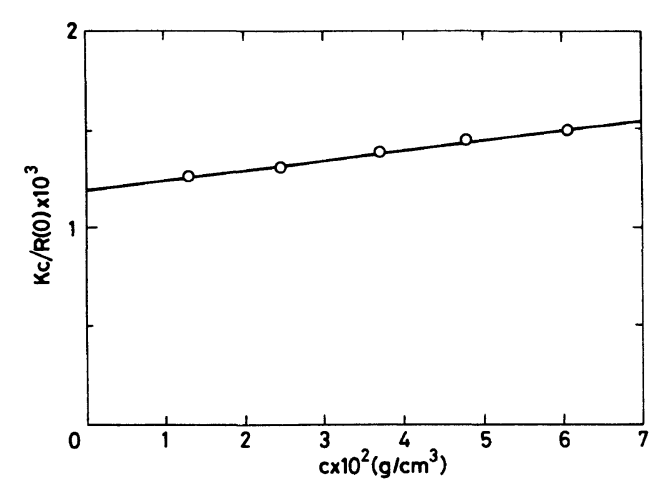

Figure 5. Concentration dependence of $K c / R(0)$ for OEG18 in acetonitrile at $25^{\circ} \mathrm{C}$, where $R(0)$ is the Rayleigh ratio at zero angle $(q=0)$.

\section{of $\mathrm{C}-\mathrm{O}$ bond.}

Since Bailey and Callard's work, ${ }^{19}$ many experiments on the characteristic ratio of PEO have been carried out, especially by viscosity measurements for various solutions. In early times, a somewhat small value of $C_{\infty}=4.0$ was proposed. ${ }^{6}$ However, recent results show that the values of $C_{\infty}$ ranges from 5.1 to 5.7. ${ }^{28}$ More recently, Abe et al. ${ }^{7}$ calculated $C_{\infty}$ with the rotational isomeric state approximation and found that the value of $C_{\infty}=5.2$ is favorably reproduced by their calculations. Our value of $C_{n}$ is consistent with Abe et al.'s value, because $C_{n}$ for finite $n$ is expected to be smaller than $C_{\infty}{ }^{6}$ Contrary to the results mentioned above, recent SANS experiments give large values for $C_{\infty}$; Kugler et al. ${ }^{21}$ obtained $C_{n}=6.9$ for in molten state and Vennemann et al.'s ${ }^{22}$ results gave a still larger value of $C_{n}=7.6$. At the present, we do not argue about the large difference between our SAXS and the SANS results.

Next, we evaluated $M_{w}$ and $A_{2}$ of OEG18 in acetonitrile from the light scattering data. To obtain these parameters, the Rayleigh ratio $R(0)$ was plotted against concentration $c$ as shown in Figure 5, where a linear relationship is observed between $K c / R(0)$ and $c$. The intercept and slope of this straight line in Figure 5 give $M_{w}=833$ and $A_{2}=2.45 \times 10^{-3} \mathrm{~mol} \mathrm{~cm}^{3}$ $\mathrm{g}^{-2}$, respectively. The observed value of $M_{w}$ is in good agreement with the expected molecular weight $M=810.9$ calculated from the chemical structure of OEG18. This agreement also confirms that OEG18 disperses molecularly in the solution as already mentioned above. The value of $A_{2}$ has also been estimated from the SAXS data; assuming $M_{w}$ of the sample as $M_{w}=810.9$ and then calculating the absolute intensity of $\Delta I(0)$, we obtained $A_{2}=2.2 \times 10^{-3}$ mol cm $\mathrm{cm}^{3}$, in good agreement with that obtained by the light scattering experiment. The values of $A_{2}$ for PEO in acetonitrile were measured by Elias and $\mathrm{Lys}^{2}$ for various molecular weights. Our values of $A_{2}$ are somewhat smaller than that estimated from their data $A_{2} \simeq 3.5 \times 10^{-3} \mathrm{~mol} \mathrm{~cm}^{3} \mathrm{~g}^{-2}$.

\section{REFERENCES}

1. F. E. Bailey, Jr. and J. V. Koleske, "Poly(ethylene oxide)," Academic Press, New York, N.Y., 1976.

2. H.-G. Elias and H. Lys, Makromol. Chem., 92, 1 (1966).

3. C. Strazielle, Makromol. Chem., 119, 50 (1968).

4. C. Cuniberti, Eur. Polym. J., 10, 1175 (1974).

5. W. F. Polik and W. Burchard, Macromolecules, 16, 978 (1983).

6. P. J. Flory, "Statistical Mechanics of Chain Molecules," Interscience, New York, N. Y., 1969.

7. A. Abe, K. Tasaki, and J. E. Mark, Polym. J., 17, 883 (1985).

8. S. Kinugasa and S. Hattori, J. Natl. Chem. Lab. Ind., 85, 129 (1990).

9. H. Utiyama, "Light Scattering from Polymer Solutions," M. B. Huglin, Ed., Academic Press, London, 1972, Chapter 4; T. Kamata and H. Nakahara, Bull. Chem. Soc. Jpn., 52, 2460 (1979).

10. H. H. Teo, R. H. Mobbs, and C. Booth, Eur. Polym. J., 18, 541 (1982).

11. E. R. Pike, W. R. M. Pomeroy, and J. M. Vaughan, J. Chem. Phys., 62, 3188 (1975).

12. B. L. Johnson and J. Smith, "Light Scattering from Polymer Solutions," M. B. Huglin, Ed., Academic Press, London, 1972, Chapter 2.

13. H. Hayashi, F. Hamada, S. Suehiro, N. Masaki, T. Ogawa, and H. Miyaji, J. Appl. Crystallogr., 21, 330 (1988).

14. T. Norisuye and H. Fujita, Polym. J., 14, 143 (1982).

15. H. Yamakawa, and J. Shimada, J. Chem. Phys., 83, 2607 (1985).

16. H. Yamakawa, Ann. Rev. Phys. Chem., 35, 23 (1984). 
17. T. Konishi, T. Yoshizaki, T. Saito, Y. Einaga, and H. Yamakawa, Macromolecules, 23, 290 (1990).

18. H.-G. Elias, Makromol. Chem., 103, 214 (1967).

19. F. E. Bailey and R. W. Callard, J. Appl. Polym. Sci., 1, 56 (1959).

20. D. R. Beech and C. Booth, J. Polym. Sci. A-2, 7, 575 (1969); E. A. Boucher and P. M. Hines, J. Polym.
Sci., Polym. Phys. Ed., 16, 501 (1978); M. Ataman and E. A. Boucher, ibid., 20, 1585 (1982).

21. J. Kugler, E. W. Fischer, M. Peuscher, and C. D. Eisenbach, Makromol. Chem., 184, 2325 (1983).

22. N. Vennemann, M. D. Lechner, and R. C. Oberthur, Polymer, 28, 1738 (1987). 